\title{
ORDER OF RESIDUAL GENERATORS - BOUNDS AND ALGORITHMS
}

\author{
Erik Frisk
}

\author{
Department of Electrical Engineering \\ Linköping University \\ Sweden \\ email:frisk@isy.liu.se
}

\begin{abstract}
This contribution analyses residual generators that perfectly decouples disturbances in linear systems. The analysis focuses on the orders of the residual generators and it is shown how low order, local relationships in the model can be utilized to increase robustness properties. Easily computed bounds on minimum and maximum order residual generators are derived and presented. An upper bound on the minimal row-degree is derived and is given directly by the number of measurements, the number of linearly independent disturbances, and the number of states in the model. A lower bound is given by the minimum observability index of the model. An upper bound for the maximum order is the number of states in the model. Copyright (C) 2000 IFAC
\end{abstract}

Keywords: fault diagnosis, residual generation, minimal indices, Kronecker indices, minimal order, disturbance decoupling

\section{INTRODUCTION}

This paper deals with supervision, or fault diagnosis, of computer controlled systems. The task of fault diagnosis is to, from known signals, i.e. measurements and control signals, detect and locate any faults acting on the system being supervised. A fundamental part of a model based diagnosis system is the residual generator. The residual generator filters known signals and generates a signal, the residual, that should be small (ideally 0) in the fault-free case and large when a fault is acting on the system. This signal can then be used as a fault indicator, signaling a faulty system.

To be able to produce a correct diagnosis in all operating conditions, influence from disturbances on the residual need to be decoupled. Also, to facilitate fault isolation, not only disturbances need to be decoupled, but also a subset of the faults. By generating a set of such residuals where different subsets of faults are decoupled in each residual, fault isolation is possible. With this approach, the design of a residual generator becomes a decoupling problem. This is the main idea behind the well used concept of structured residuals (Gertler 1991).

This work is a study of the complexity of linear residual generators for linear systems with no model uncertainties where any faults and disturbances acting on the sys- tem are modeled as input signals. Of particular interest is the minimum complexity of residual generators.

\section{LOW ORDER RESIDUAL GENERATORS}

The reason for the interest in the low and minimal order properties of the residual generator is primarily that we want to depend on the model as little as possible. A low order usually implies that only a small part of the model is utilized, i.e. local relationships in the model is utilized. Since all parts of the model have errors, this further means that few model errors will affect the residual. Also, lower complexity of the residual generator means easier implementation and less on-line computational burden. The following small example will highlight this issue. Consider a linear system with two sensors, one actuator, and a modeled sensor fault in the second sensor.

$$
\left(\begin{array}{l}
y_{1} \\
y_{2}
\end{array}\right)=\left(\begin{array}{c}
\frac{1}{s+a} \\
\frac{1}{(s+b)(s+a)}
\end{array}\right) u+\left(\begin{array}{l}
0 \\
1
\end{array}\right) f
$$

The model consists of two model parameters, $a$ and $b$. To detect the fault, a second order residual 


$$
r_{1}=y_{2}-\frac{1}{(s+a)(s+b)} u
$$

can be used. Examining the expression gives that the residual relies on the accuracy of both model parameters $a$ and $b$. Using straightforward manipulations of model equations, it is possible to derive a new, first order residual representing a local relationship between the two sensor signals:

$$
r_{2}=\frac{1}{s+b} y_{1}-y_{2}
$$

As can be seen, residual $r_{2}$ only depends on the accuracy of parameter $b$. Thus, a lower order residual generator resulted in a residual generator less dependent on the model accuracy. Here, in this example, even complete invariance of model accuracy of parameter $a$ was achieved. This is not a general result, model dependency does not always decrease with the order. However, if the model has such a property, systematic utilization of low-order residual generators is desirable.

\section{PRELIMINARIES}

This paper relies on established theory on polynomial matrices, rational vector spaces, and polynomial bases for these spaces (Kailath 1980, Forney 1975, Chen 1984). The main notions used, are presented in this section.

The row-degree of a row vector of polynomials is defined as the largest polynomial degree in the row-vector. In this paper, polynomial bases and orders of polynomial bases are of special interest. A polynomial basis is here represented by a polynomial matrix where the rows are the basis vectors. The order of a polynomial basis $F(s)$ is defined as the sum of the its row-degrees. A minimal polynomial basis for a rational vector-space $\mathcal{F}$ is then any polynomial basis that minimizes this order.

A matrix $F(s)$ is irreducible if and only if $F(s)$ has full rank for all $s$. Any matrix $F(s)$ can always be written as

$$
F(s)=S(s) D_{h r}+L(s)
$$

where $S(s)=\operatorname{diag}\left\{s^{\mu_{i}}, i=1, \ldots, p\right\}, D_{h r}$ is the highestrow-degree coefficient matrix, $\mu_{i}$ is the row-degrees, and $L(s)$ is the rest term with row degrees strictly less than $\mu_{i}$. A matrix is row-reduced if its highest row-degree coefficient matrix $D_{h r}$ has full row rank.

In addition to these definitions, the following theorems will be used:

Theorem 1. (Kailath,1980, Theorem 6.5-10). The rows of a matrix $F(s)$ form a minimal polynomial basis for the rational vector space they generate, if and only if $F(s)$ is irreducible and row-reduced.

Theorem 2. (Kailath,1980). For any linear matrix pencil $A-s B$, it is possible to find constant, square, and nonsingular matrices $U$ and $V$ such that

$$
\begin{aligned}
& U(A-s B) V= \\
= & \text { block-diag }\left\{L_{\mu_{1}}, \ldots, L_{\mu_{\alpha}}, \widetilde{L}_{\nu_{1}}, \ldots, \widetilde{L}_{\nu_{\beta}}, s J-I, s I-F\right\}
\end{aligned}
$$

where

(1) $F$ is in Jordan form

(2) $J$ is a nilpotent Jordan matrix
(3) $\widetilde{L}_{\nu}$ is a $(\nu+1) \times \nu$ matrix of the form

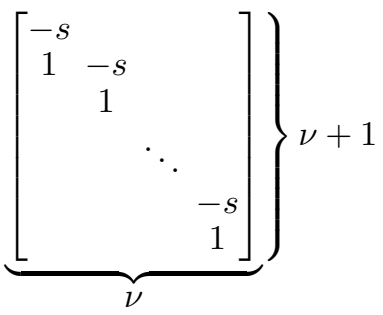

(4) $L_{\mu}=\widetilde{L}_{\mu}^{T}$

The $\left\{\nu_{i}\right\}$ and $\left\{\mu_{i}\right\}$ are called left and right Kronecker indices and are of particular interest in this paper.

Note: All matrices, besides $\widetilde{L}_{\nu}$ have full row-rank, $\widetilde{L}_{\nu}$ will therefore characterize the left null-space structure of the pencil. It is also easy to check that the left nullspace of $\widetilde{L}_{\nu}$ is given by

$$
v(s)=\left[\begin{array}{llll}
1 & s & \cdots & s^{\nu}
\end{array}\right]
$$

i.e, the degree of the (left) null-space vectors is directly given by the (left) Kronecker indices.

\section{LINEAR RESIDUAL GENERATION}

This section is a brief presentation of the linear residual generation problem. All derivations are performed in the continuous-time case but the corresponding results for the discrete-time case can be obtained by substituting $\mathrm{s}$ by $\mathrm{z}$ and improper by non-causal.

The systems studied in this work are assumed to be on the form

$$
y=G_{u}(s) u+G_{d}(s) d+G_{f}(s) f
$$

where $y$ is the measurement vector, $u$ is the vector of known inputs to the system, $d$ is the disturbance vector, and $f$ is the vector of faults. The symbols $k_{u}, k_{d}, k_{f}$, and $m$ will be used to denote the number of known inputs, disturbances, faults, and measurements respectively. A general linear residual generator can be written

$$
r=Q(s)\left(\begin{array}{l}
y \\
u
\end{array}\right)
$$

Here, $Q(s)$ is a single output filter that is said to be a residual generator if and only if $r=0$ for all $d$ and $u$ when $f=0$. To be able to detect faults, it is also required that $r \neq 0$ when $f \neq 0$.

Inserting (1) into (2) gives

$$
r=Q(s)\left[\begin{array}{cc}
G_{u}(s) & G_{d}(s) \\
I & 0
\end{array}\right]\left[\begin{array}{l}
u \\
d
\end{array}\right]+Q(s)\left[\begin{array}{c}
G_{f}(s) \\
0
\end{array}\right] f
$$

To make $r(t)=0$ when $f(t)=0$, it is required that disturbances and the control signal are decoupled, i.e. for $Q(s)$ to be a residual generator, it must hold that

$$
Q(s)\left[\begin{array}{cc}
G_{u}(s) & G_{d}(s) \\
I & 0
\end{array}\right]=0
$$

This implies that $Q(s)$ must belong to the left null-space of

$$
M(s)=\left[\begin{array}{cc}
G_{u}(s) & G_{d}(s) \\
I & 0
\end{array}\right]
$$

This null-space is denoted $\mathcal{N}_{L}(M(s))$. Therefore, the matrix $Q(s)$ need to fulfill two requirements: belong 
to the left null-space of $M(s)$ to ensure disturbance decoupling and have good fault sensitivity properties. In (Nyberg \& Frisk 1999), a design procedure based on finding a basis for all rational $Q(s) \in \mathcal{N}_{L}(M(s))$ is described in detail. A main result on how to compute this basis is repeated in Section 4.1. In this paper, it is assumed that such a basis can be computed and is denoted $N_{M}(s)$. It is noteworthy that, by inspection of (3), it holds that the dimension (number of rows) of $N_{M}(s)$ is less than or equal to the number of measurements.

The residual generator $Q(s)$ can, after the null-space basis $N_{M}(s)$ is computed, be formed as

$$
Q(s)=c^{-1}(s) \phi(s) N_{M}(s)
$$

where $\phi(s)$ is a polynomial row-vector and $c(s)$ is a scalar polynomial. The matrices $\phi(s)$ and $c(s)$ includes all design freedom available to the designer, e.g. the dynamics of the residual generator. The only constraint on $c(s)$, apart from stability constraints, is a degree constraint. The degree of $c(s)$ must be larger or equal to the row-degree of $\phi(s) N_{M}(s)$ to make the residual generator realizable. This also means that the minimal order of a realization of a decoupling filter is determined by the row-degrees of the minimal polynomial basis $N_{M}(s)$.

\subsection{Computing the Basis}

A main result in computing the basis $N_{M}(s)$ is to reduce the problem of computing a minimal polynomial basis for the left null-space of the rational matrix $M(s)$ into the problem of finding a minimal polynomial basis for the left null-space of a polynomial matrix. This is then a standard problem in the theory of polynomial matrices for which there exists standard tools readily available (The Polynomial Toolbox 2.0 for Matlab 5 1998).

The results of this section assumes that the system is written in state-space form

$$
\begin{aligned}
& \dot{x}=A x+B_{u} u+B_{d} d \\
& y=C x+D_{u} u+D_{d} d
\end{aligned}
$$

and $n$ will be used to denote the number of states of such a minimal realization.

Then an important matrix, which will be used extensively, the system matrix in state-space form with disturbances as inputs, can be formed. This matrix is denoted $M_{s}(s)$ and looks like:

$$
M_{s}(s)=\left[\begin{array}{cc}
C & D_{d} \\
-(s I-A) & B_{d}
\end{array}\right]
$$

Also, the following matrix, denoted $P$, will be used:

$$
P=\left[\begin{array}{cc}
I_{m} & -D_{u} \\
0_{n \times m} & -B_{u}
\end{array}\right]
$$

Then, the main theorem used here can be stated as:

Theorem 3. (Frisk,1998;Nyberg,1999). Let $V(s)$ be a minimal polynomial basis for $N_{L}\left(M_{s}(s)\right)$ and let the pair $\left\{A,\left[\begin{array}{ll}B_{u} & B_{d}\end{array}\right]\right\}$ be controllable. Then it holds that $W(s)=V(s) P$ is a minimal polynomial basis for $M(s)$.

Remark 1: This theorem shows how the system matrix $M_{s}(s)$ is central in computing and analyzing the basis $N_{M}(s)$ and motivates its use in subsequent sections.
Remark 2: Since $N_{M}(s)=N_{M_{s}}(s) P$ where $P$ is a constant matrix, it is clear that the the row-degrees of basis $N_{M}(s)$ is less or equal to the row-degrees of $N_{M_{s}}(s)$. In the next section, this observation will be strengthened and it is proved that the row-degrees of $N_{M}(s)$ and $N_{M_{s}}(s)$ are in fact equal.

\section{ROW-DEGREES OF BASIS}

As discussed in the previous section, the row-degrees of a minimal polynomial basis for $\mathcal{N}_{L}(M(s))$ is closely connected to the order of the analytical relation used in the residual generator, and also the order needed to implement the residual generator. In this section, easily computed bounds on the minimal and maximal rowdegrees of the basis $N_{M}(s)$ are derived.

Before further analysis on row degrees is made, a lemma is needed that shows that examining row-degrees of a basis for the relatively unstructured matrix $M(s)$ can be performed by examining the row degrees of the, structurally, much simpler system matrix $M_{s}(s)$. The primary property of $M_{s}(s)$ that makes it suitable for analysis is the fact that it has degree 1 , i.e. it is a matrix pencil.

Lemma 4. The row-degrees of a minimal polynomial basis for $\mathcal{N}_{L}(M(s))$ is equal to the row-degrees of a minimal polynomial basis for $\mathcal{N}_{L}\left(M_{s}(s)\right)$, where $M_{s}(s)$ is the system matrix with the pair $\left\{A,\left[B_{u} B_{d}\right]\right\}$ controllable.

\section{PROOF.}

Let $V(s)$ be a minimal polynomial basis for $\mathcal{N}_{L}\left(M_{s}(s)\right)$ and partition $V(s)=\left[V_{1}(s) V_{2}(s)\right]$ according to the partition of $M_{s}(s)$. Since $V(s) \in \mathcal{N}_{L}\left(M_{s}(s)\right)$, it holds that

$$
V_{1}(s) C=V_{2}(s)(s I-A)=s V_{2}(s)-V_{2}(s) A
$$

Also, since each row degree of $s V_{2}(s)$ is strictly greater than the corresponding row-degree of $V_{2}(s) A$, it holds that for each row $i$

$$
\begin{aligned}
& \text { row-deg }{ }_{i} s V_{2}(s)=1+\text { row- } \operatorname{deg}_{i} V_{2}(s)= \\
& =\text { row- }_{-} \operatorname{deg}_{i} V_{1}(s) C
\end{aligned}
$$

The above equation can be rearranged to

$$
\text { row-deg } V_{2}(s)<\text { row-deg }_{i} V_{1}(s) C \leq \text { row-deg }_{i} V_{1}(s)
$$

i.e. row- $\operatorname{deg}_{i} V(s)=$ row- $\operatorname{deg}_{i} V_{1}(s)$. From the definition of $P$ it follows that

$$
\begin{aligned}
& W(s)=\left[W_{1}(s) W_{2}(s)\right]=V(s) P= \\
& =\left[\begin{array}{ll}
V_{1}(s) & \left(-V_{1}(s) D_{u}-V_{2}(s) B_{u}\right)
\end{array}\right]
\end{aligned}
$$

Equations (7) and (8) directly give

$$
\text { row-deg } W(s)=\text { row- }_{i} \operatorname{deg}_{i} V_{1}(s)=\operatorname{row}_{-} \operatorname{deg}_{i} V(s),
$$

i.e. the row degrees of $W(s)$ and $V(s)$ are equal. According to Theorem $3, W(s)$ and $V(s)$ are minimal polynomial bases for $\mathcal{N}_{L}(M(s))$ and $\mathcal{N}_{L}\left(M_{s}(s)\right)$ respectively and the lemma follows immediately.

Much of the structure of a matrix pencil is revealed by the Kronecker Canonical Form, given by Theorem 2. Specifically, the degrees of $N_{M_{s}}(s)$, and according to Lemma 4 the degrees of $N_{M}(s)$, is directly given by 
the left Kronecker indices ${ }^{1}$, which can be extracted directly from a pencil on KCF. However, transferring a general pencil to KCF is a numerically tricky operation. It is therefore desired to have easily computed bounds or numerically stable algorithms for calculating these indices. In Section 5.1, bounds for the minimum and maximum row-degree of $N_{M}(s)$ are given. Section 5.2 gives pointers to some algorithms, that can be used to calculate the row-degrees without actually computing the basis.

\subsection{Bounds on row-degrees}

This section primarily analyzes the minimal row-degree $\rho_{\text {min }}$, of the basis, since $\rho_{\text {min }}$ is closely connected to the minimum complexity of a residual generator. However, before bounds on the minimum row-degree is derived, an upper bound on all row-degrees of a basis is directly given by the following theorem:

Theorem 5. (Nyberg,1999). A matrix whose rows form a minimal polynomial basis for $\mathcal{N}_{L}(M(s))$ has all rowdegrees $\leq n$.

Now, an upper and a lower bound on the minimum rowdegree is derived. First, a lower bound is derived, given by the following theorem:

Theorem 6. A lower bound for the minimal row-degree $\rho_{\text {min }}$ of a basis for $\mathcal{N}_{L}(M(s))$ is given by the minimal observability index of the pair $(A, C)$.

For the proof of this theorem, and other theorems to follow, the following lemma is needed. Denote

$$
\widetilde{M}_{\rho}=\underbrace{\left[\begin{array}{ccccc}
Q & R & & & \\
& Q & R & & \\
& & \ddots & \ddots & \\
& & Q & R
\end{array}\right]}_{(\rho+2)\left(n+n_{d}\right)}\}(\rho+1)(m+n)
$$

where $M_{s}(s)=Q+s R$ and $Q, R$ are constant matrices. Then,

Lemma 7. The space $\mathcal{N}_{L}\left(M_{s}(s)\right)$ contains a $\rho$-degree polynomial vector if and only if $\widetilde{M}_{\rho}$ does not have full row rank.

PROOF. Let $F(s)$ be a $\rho$-degree polynomial matrix in $\mathcal{N}_{L}\left(M_{s}(s)\right)$. Then it holds that

$$
\begin{aligned}
0=F(s) M_{s}(s)= & \left(F_{0}+F_{1} s+\cdots+s^{\rho} F_{\rho}\right) M_{s}(s)= \\
= & {\left[\begin{array}{lll}
F_{0} & F_{1} \cdots F_{\rho}
\end{array}\right]\left[\begin{array}{c}
M_{s}(s) \\
s M_{s}(s) \\
\vdots \\
s^{\rho} M_{s}(s)
\end{array}\right]=\widetilde{F} \widetilde{M}_{\rho}\left[\begin{array}{c}
I \\
s I \\
\vdots \\
s^{\rho} I
\end{array}\right] }
\end{aligned}
$$

From the equation above it is clear that a $\rho$-degree polynomial $F(s)$ is in $\mathcal{N}_{L}\left(M_{s}(s)\right)$ if and only if $\widetilde{F} \widetilde{M}_{\rho}=$ 0 . The lemma follows directly because such a $\widetilde{F}$ can only exist if $\widetilde{M}_{\rho}$ does not have full row-rank.

\footnotetext{
1 The Kronecker indices is sometimes called minimal indices.
}

Return to the proof of Theorem 6 .

PROOF. Denote the system matrix without disturbances with $M_{s}^{(n d)}(s)$, i.e.

$$
M_{s}^{(n d)}(s)=\left[\begin{array}{c}
C \\
s I-A
\end{array}\right]
$$

It is well known (Kailath 1980, p. 413), that the rowdegrees of a minimal polynomial basis for the left nullspace of $M_{s}^{(n d)}(s)$ is equal to the observability indices of the pair $(A, C)$. Let $c_{\text {min }}$ be the minimum observability index of $(A, C)$. Then, according to Lemma $7, c_{\min }$ is the lowest $\rho$ such that $\widetilde{M}_{\rho}^{(n d)}$ does not have full row-rank. Let

$$
\begin{aligned}
& Q=\left[\begin{array}{ll}
Q_{1} & Q_{2}
\end{array}\right]=\left[\begin{array}{ll}
C & D_{d} \\
A & B_{d}
\end{array}\right] \\
& R=\left[\begin{array}{ll}
R_{1} & R_{2}
\end{array}\right]=\left[\begin{array}{cc}
0 & 0 \\
-I & 0
\end{array}\right]
\end{aligned}
$$

Then, by a trivial column reordering, $\widetilde{M}_{\rho}$ can be written on the form

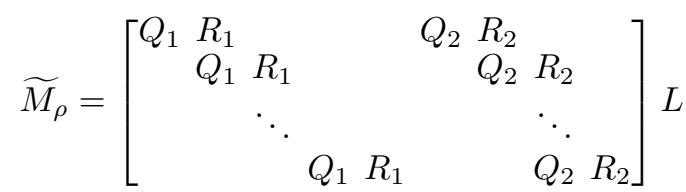

$$
\begin{aligned}
& =\left[\widetilde{M}_{\rho}^{(n d)} \star\right] L
\end{aligned}
$$

where $L$ is a square, full rank pivoting matrix and $\star$ is any matrix. From the equation above, it is clear that if $\widetilde{M}_{\rho}^{(n d)}$ has full row-rank, then also $\widetilde{M}_{\rho}$ has full row-rank. Also, for all $\rho<c_{\min }, \widetilde{M}_{\rho}^{(n d)}$ and thereby also $\widetilde{M}_{\rho}$, will have full row-rank. The theorem then follows directly from Lemma 7 , i.e. there exists no $\rho$-degree polynomial in $\mathcal{N}_{L}\left(M_{s}(s)\right)$ where $\rho<c_{\min }$.

Remark: This result can also be found, without proof, in (Ding, Ding \& Jeinsch 1998).

Theorem 8. An upper bound for the minimal rowdegree $\rho_{\text {min }}$ of a basis for $\mathcal{N}_{L}(M(s))$ is given by

$$
\rho_{\min } \leq\left\lfloor\frac{n+n_{d}}{m-n_{d}}\right\rfloor
$$

where

$$
n_{d}=\operatorname{Rank}\left(\begin{array}{c}
B_{d} \\
D_{d}
\end{array}\right)
$$

is the number of linearly independent disturbances. The $\lfloor\cdot\rfloor$ operator is the floor operation.

PROOF. Let $A, B_{u}, B_{d}, D_{u}$, and $D_{d}$ be a minimal state-space realization of $\left[G_{u}(s) G_{d}(s)\right]$. If $n_{d}<k_{d}$, i.e. there exist linear dependencies between disturbances, rewrite the system description with a new set of $n_{d}$ linearly independent disturbances. That is, find $\widetilde{B}_{d}$ and $\widetilde{D}_{d}$ with dimensions $n \times n_{d}$ and $m \times n_{d}$ respectively so that

$$
\operatorname{Im}\left(\begin{array}{c}
B_{d} \\
D_{d}
\end{array}\right)=\operatorname{Im}\left(\begin{array}{c}
\widetilde{B}_{d} \\
\widetilde{D}_{d}
\end{array}\right)
$$

and use these in the state-space description. Now, using Lemma 4 and 7 it is clear that a $\rho$-degree polynomial vector is in $\mathcal{N}_{L}(M(s))$ if and only if $\widetilde{M}_{\rho}$ does not have 
full row rank. A sufficient condition for $\widetilde{M}_{\rho}$ not to have full row-rank, is that the number of rows is larger than the number of columns, i.e.

$$
(\rho+1)(m+n)>(\rho+2)\left(n+n_{d}\right)
$$

Straightforward manipulations of the inequality results in

$$
\rho>\frac{n+n_{d}}{m-n_{d}}-1
$$

Note that $m-n_{d}>0$ is a necessary condition for the existence of a residual generator. Therefore, the smallest integer $\rho$ that fulfills the inequality is $\left\lfloor\frac{n+n_{d}}{m-n_{d}}\right\rfloor$ which completes the proof.

Remark: A similar result without disturbance decoupling, i.e. $n_{d}=0$, can be found in (Mironovskii 1980).

The result of Theorem 8 is useful when selecting the set of faults that are to be decoupled in the residual, i.e. when shaping the fault isolation properties. This theorem gives direct access to information on the expected complexity of the resulting residual generator, thereby making it possible to estimate the complexity of all residual generators without actually performing the designs. When shaping the isolation structure, i.e. selecting which and how many faults that are to be decoupled in each residual, the designer controls the quantity $m-n_{d}$. It holds that

$$
\frac{n+n_{d}}{m-n_{d}}=\frac{n+m}{m-n_{d}}-1
$$

i.e. the upper bound decreases as $1 / x$ with the designer controlled quantity $m-n_{d}$. Since the decrease is quite rapid, the complexity gain can be substantial, especially for high order processes that are well equipped with sensors.

\subsection{Calculation of row-degrees}

It is well known (Kailath 1980, p. 413), that the rowdegrees of a minimal polynomial basis for the left nullspace of $M_{s}^{(n d)}(s)$ equal the observability indices of the pair $(A, C)$. However, no such straightforward algorithm for computing the minimal indices exists for the general case including disturbances.

Of course, one could calculate the basis as described in Section 4. However, there are reasons for computing the indices without actually computing the basis itself. By only computing the Kronecker indices, which is a smaller problem than actually computing the basis, it is reasonable to assume that this would pose a numerically easier problem ${ }^{2}$.

There exist a lot of literature and algorithms for computing the Kronecker indices of a general pencil, e.g. (Misra, Dooren \& Varga 1994, Wang, Dorato \& Davison 1975, Aling \& Scumacher 1984, Kågström 1986).

\section{EXAMPLES}

This section contains one small example where the results are applied, followed by a discussion for a larger industrial application, a model of a military jet-engine.

\footnotetext{
2 To the authors knowledge, no such investigation has been made.
}

\section{Small Example}

Consider a system given by the following transfer functions.

$y=G_{u}(s) u+G_{d}(s) d=\left[\begin{array}{c}\frac{1}{s+1} \\ \frac{1}{s+2} \\ 0\end{array}\right] u+\left[\begin{array}{c}\frac{2}{s+4} \\ \frac{2}{s+4} \\ \frac{3 s+12}{(s+4)(s+3)}\end{array}\right] d$

This model can be realized by a 4 :th order state-space description, i.e. $n=4$. Matrix $M(s)$ then becomes

$$
M(s)=\left(\begin{array}{cc}
\frac{1}{s+1} & \frac{2}{s+4} \\
\frac{1}{s+2} & \frac{2}{s+4} \\
0 & \frac{3 s+12}{(s+4)(s+3)} \\
1 & 0
\end{array}\right)
$$

Using Theorems 5,6, and 8 give that for the maximum and minimum row-degrees $\rho_{\max }$ and $\rho_{\min }$ it holds that

$$
\begin{aligned}
1 \leq \rho_{\min } & \leq 2 \\
\rho_{\max } & \leq 4
\end{aligned}
$$

The lower bound on $\rho_{\min }$ is given by the observability indices of the pair $(A, C)$ which can be calculated to $2,1,1$. Computing a basis gives

$$
N_{M}(s)=\left[\begin{array}{cccc}
-s^{2}-3 s-2 & s^{2}+3 s+2 & 0 & 1 \\
3 s+3 & -4.5 s-9 & s+3 & 1.5
\end{array}\right],
$$

i.e. $\rho_{\min }=1$ and $\rho_{\max }=2$, which confirms that the inequalities (9) holds.

\section{Jet-Engine Model}

A model of a jet-engine developed by Volvo Aero Corporation, Trollhättan, Sweden, is used in this example. A high-order non-linear model of the engine is used for analysis and control design. This model can also be used for diagnosis purposes. The model was linearized in an operating point and the resulting model, after that noncontrollable and non-observable modes are eliminated, is a 26:th order model. The model includes 8 sensors and 4 actuators.

It turns out, due to physical reasons, that the model is stiff with a $10^{5}$ ratio between largest and smallest time-constant. This model stiffness together with the high-order makes the model numerically sensitive which demands good numerical properties of the design algorithm.

In the design example, faults in sensors and actuators are considered. A residual that indicates a sensor failure is to be designed, i.e. all 4 actuator faults are to be decoupled. Using Theorem 8, it is clear that there exists residual generators with degree less than $\left\lfloor\frac{26+4}{8-4}\right\rfloor=7$, which is significantly less than system order. Worth noting is how this limit depends on $n_{d}$. If a residual were to be designed that decoupled only one fault, i.e. $n_{d}=1$, then the upper bound on the minimum degree residual generator would be as low as 3 . This shows how it is possible to trade isolation properties for simpler fault detection filters. 
It is also worth noting that, a design method not considering the order of the resulting residual generator easily results in a residual generator of the same order as the process model, here 26 . However, with the minimal polynomial basis approach presented in Section 4, a 4:th order residual generator was found which shows how the minimality property here results in a filter with substantially less order than the order of the design model.

As mentioned, this model poses a numerically difficult design problem. Calculating the row-degrees of the basis with three different methods resulted in three different sets of row-degrees according to the table below:

\begin{tabular}{l|l} 
Method & row-degrees of basis \\
\hline \hline (Wang et al. 1975) & $\{3,3,4,4\}$ \\
null command & $\{4,4,4,4\}$ \\
pennull command & $\{5,6,6,6\}$
\end{tabular}

The null command is included in (The Polynomial Toolbox 2.0 for Matlab 5 1998). The pennull command uses an algorithm, especially developed for extracting null-spaces for matrix pencils and is based on (Beelen \& Weltkamp 1987, Dooren 1979).

When evaluating the obtained basis by multiplying $N_{M}(s) M(s)$, the product does not become exactly 0 due to finite precision arithmetic. It does however become close to zero, but the row-degrees does not match the degrees obtained with the method described in (Wang et al. 1975).

\section{CONCLUSIONS}

This contribution analyses residual generators that perfectly decouple disturbances in linear systems. Specifically, the possible orders of residual generators is analyzed given a system model.

One main contribution is the derivation of easily computed bounds for the minimal order residual generator. Of special interest is minimum and low order residual generators. It is described how low-order consistency relations, of significantly lower order than process order, can represent local relationships between sensor or actuator signals. Thus, basing the residual generator design on those local relationships, the residual generator only depends on the model parameters in that relationship. Thus, utilizing local relationships can introduce robustness properties.

An upper bound on the minimal row-degree is derived and given directly by the number of measurements, the number of linearly independent disturbances, and the number of states in the model. A lower bound is given by the minimum observability index of the model.

These bounds can help the designer to estimate complexity of the diagnosis system and also help to guide the numerical algorithms used to find solutions to the residual generation problem.

Design examples are included to illustrate the use of the bounds. A design is performed on a 26:th order model of a jet-engine. A design algorithm that does not explicitly address minimality issues will likely end up with a residual generator of the same order as the system model. With the derived bounds, it was clear that a $7:$ th order residual generator existed. Performing the design with the proposed algorithm, a 4:th order residual generator was found.

\section{ACKNOWLEDGMENTS}

The PolyX, Ltd. team in general and Dr. Didier Henrion and Dr. Michael Sebek in particular are greatfully acknowledged for implementing the pennull algorithm.

\section{REFERENCES}

Aling, H. \& Scumacher, M. (1984). A nine-fold canonical decomposition for linear systems, International Journal of Control 39(4): 779-805.

Beelen, T. \& Weltkamp, G. (1987). Numerical computation of a coprime factorization of a transfer function matrix, Systems and Control Letters 9: 281-288.

Chen, C.-T. (1984). Linear System Theory and Design, Holt, Rinehart and Winston, New York.

Ding, S., Ding, E. \& Jeinsch, T. (1998). A numerical approach to analysis and design of FDI systems, Proc. of. IEEE Conf. on Decision and Control, pp. $1155-1160$.

Dooren, P. (1979). The computation of kronecker's canonical form of a singular pencil, Linear Algebra and its Applications 27: 103-140.

Forney, G. (1975). Minimal bases of rational vector spaces, with applications to multivariable linear systems, SIAM J. Control 13(3): 493-520.

Frisk, E. (1998). Residual Generation for Fault Diagnosis: Nominal and Robust Design, Licentiate thesis LIU-TEK-LIC-1998:74, Linköping University.

Gertler, J. (1991). Analytical redundancy methods in fault detection and isolation; survey and synthesis, IFAC Fault Detection, Supervision and Safety for Technical Processes, Baden-Baden, Germany, pp. 9-21.

Kailath, T. (1980). Linear Systems, Prentice-Hall.

Kågström, B. (1986). RGSVD - an algorithm for computing the kronecker structure and reducing subspaces of singular $A-\lambda B$ pencils, SIAM J. Sci. Stat. Comput 7(1): 185-211.

Mironovskii, L. (1980). Functional diagnosis of linear dynamic systems, Automation and Remote Control pp. 1198-1205.

Misra, P., Dooren, P. \& Varga, A. (1994). Computation of structural invariants of generalized state-space systems, Automatica 30(12): 1921-1936.

Nyberg, M. (1999). Model Based Fault Diagnosis: Methods, Theory, and Automotive Engine Applications, $\mathrm{PhD}$ thesis, Linköping University.

Nyberg, M. \& Frisk, E. (1999). A minimal polynomial basis solution to residual generation for fault diagnosis in linear systems, Proc. of IFAC 1999 World Congress, Vol. P, Beijing, P.R. China, pp. 61-66.

The Polynomial Toolbox 2.0 for Matlab 5 (1998). Polyx, Czech Republic. URL: http://www.polyx.com.

Wang, S., Dorato, P. \& Davison, E. (1975). On the computation of minimal indices for linear multivariable systems, International Journal of Control 21(4): $537-543$. 